\title{
III.
}

Aus der Kgl. Universitäts-Ohrenklinik zu Halle a. S.

\section{Geheilter Fall von Pyaemia ex otitide; Unterbindung der Vena jugularis; Durchspülung ihres peripheren Endes und des Sinus transversus.}

\author{
Von \\ Dr. Grunert, \\ I. Assistenzarzt der Thinik.
}

In der Literatur) sind im Ganzen 8 Fälle von Pyaemia ex otitide publicirt, bei welchen im Einklang mit einem von $\mathrm{Zau}$ fal im Jahre 1880 gemachten Vorschlage die Vena jugularis der ohrkranken Seite unterbunden wurde in der Absicht, auf diese Weise die Aufnalme des infectiösen Thrombenmaterials im Sinus transversus in die Blutbahn zu verhindern und somit gewissermaassen den Krankheitsherd aus dem Körper auszuschalten.

Von diesen 8 Fällen gelangten 6 zur Heilung, „ein gewiss sehr ermunterndes Resultat - wie Sehwartze sagt -, um dem Vorschlage von Za ufal weiterbin Folge zu geben".2)

Verfasser hatte Gelegenheit, in den Osterferien dieses Jahres als Vertreter des Directors der Halle'schen Obrenklinik einen weiteren hierher gehörigen Fall zu operiren, welcher das Interesse der Fachgenossen in mancher Hinsicht in Anspruch zu nebmen wohl geeignet sein dürfte.

Der Fall ist folgender:

Anna Gründler, 10 Jahre alt, Arbeiterkind aus Giebichenstein bei Halle. Seit Anfang Mărz 1893 mit der Klage über Schwerhörigkeit beiderseits, sowie über Behinderung der Nasenathmung in poliklinischer Behandiung. Diagnose: subacuter schleimiger Katarrh beiderseits und reichliche adenoide Vegetationen im Nasenrachenraum. Am 20. März Entfernung der Vegetationen mit dem Trautmann'schen scharfen Löffel. Am 21. März Klage über starken

1) Die Literatur hat Schwartze in seinem Handbuch Bd. II, S. 843 fr. zusammengestellt.

2) Schwartze's Handbuch Bd. II. S. 846 . 
Schmerz im linken Ohr, Schlaflosigkeit. Die Paracentese des stark gerötheten und in seiner hinteren Hälfte vorgewöbten Trommelfells entleerte Schleimeiter. Nach der Paracentese Aufhören der Schmerzen, am 25. März Sistiren der Eiterung. In der Nacht rom 25. zum 26. März ron Neuem heftige Schmerzen im linken Ohr. Am 26. März Schmerzen hinter dem Ohr, Anschwellung daselbst, Fieber, kein Schütelfrost, Sch indel, vollige Appetitiosigkeit. Aufnahme in die stationäre Klinik.

Status vom 26. März. Sehr anämisches Kind. Innere Organe normal. Obstipation. Beim Gehen mit gesehlossenen Augen Schwindel nach links und hinten. Pupillen gleich weit, reagiren gnt. Keine weiteren Cerebralsymptome. Anschwellung, Röthung und starke Druckemptindlichkeit hinter dem linken Ohr ohne Fluctuation.

Gehörgang nicht stenosirt, starke Röthung der Gehörgangshaut im knöchernen Theile desselben. Im Trommelfell centrale kleine Perforation mit eitrig belegten Rändern.

Hörprüfungsergebniss: Flüsterzahlen links verschärft am Ohr, rechts 4 Meter. Fis 4 links bedeutend herabgesetzt. $C_{1}$ vom Scheitel unbestimmt.

Ergebniss des Catheterismus tubae: Links Perforationsgeră usch mit Rasseln. Rechts: Rasselgeräusch.

Abendtemperatur 39,5 (s. Temperaturtabelle).

28. März. Leichte Druckempfindlichkeit im Verlauf der Vena jugularis. Abendtemperatur 39,5 .

29. März. Morgentemperatur 38,4. Somnolent, antwortet nur anf stärkeres Anrufen.

29. März. Aufmeisselung links: Schnitt durch die infltrirten Weichtheile und das verdickte Periost, welches durch Eiter von der Corticalis abgehoben ist. Letztere zeigt keinen Durchbruch. Typische Eröfinung des Antrum m., welches keinen Eiter enthält. Der Warzenfortsatz ist ausserst reich an pneumatischen Zellen, welche sämmtlich strotzend mit Eiter gefullt sind. Aus der ganz mit Eiter gefülten Spitze des Proc. mastoidens quill das Secret unter Pulsation hervor. Eine Communication mit dem Cavum cranif ist nicht nachweisbar. Drain in das Antrum gelegt, 2 Nähte, Verband.

31. März. Verbandwechsel; am Aussehen der Wunde nichts Abnormes. Vergangene Nacht Erbrechen. Die Temperatur schwankt weiter zwischen 36,0 and 40,5 .

2. April. Die Kranke klagt uber Schmerzhaftigkeit auf der Aussenseite des linken Oberschenkels, schreit bei leisester Berührung auf. Gelenke frei und schmerzlos beweglich. Die Wunde zeigt keine Neigung zur Granulationsbildung. Einzelue freiliegende Zellen zeigen eine grüngraue Verfärbung ihrer Wandung.

3. April. Die Schmerzhaftigkeit des linken Oberschenkels ist bedeutend geringer, dagegen Empfindlichkeit des rechten Eandgelenkes. Appetit gering. Somnolenz unverändert. Kein Schüttelfrost.

4. April. Status idem. Kein Eiweiss oder Zucker im Urin. Schmerzhaftigkeit der rechten Schultergegend, Jugularis nicht druckempfindich. Ordination: Wein, Chinin 0,5 , bei Steigerung der Temperatur bis $39^{\circ}$. Abends Schüttelfrost von $1 / 2$ Stunde Dauer, danach Temperatur von $40^{\circ}$. Schmerzhaftigkeit der rechten Schultergegend. 
5. April. Operative Freilegung des Sinus transversus in etwa $2: 1 \mathrm{Cm}$. Ausdehnung. Die gelblich verfärbte Wand desselben ist eitrig infiltrirt. Freilegung und Unterbindung der Vena jugularis. Das Stück derselben peripher von der Unterbindungsstelle zeigte sich thrombosirt. Es wird eröffnet, and nachdem im Sinus transversus eine Gegenöffnung angelegt war, welche die Anwesenheit von eitrig zerfallenen Thrombenmassen in demselben bestätigte, werden die Thrombenmassen aus der Jugularvene und dem Sinus transversus durch einen Irrigationsstrom mit einer 0,75 proc. Kochsalzlösung, welcher unter măssigem Druck angewandt wurde, ausgespult. Es wurde so lange irrigirt, bis der durch das Foramen jugulare laufende Wasserstrom klar aus der Incisionsöffnung des Sinus transversus wieder abfloss. Peripher von der Oeffnung im Sinus transversus erstreckte sich die Thrombose weiter; diese Thromben wurden indess nicht weiter beachtet, weil sie keinen eitrigen Zerfall zeigten. Tamponade des Sinus transversus und der Halswunde mit Jodoformgaze, am Halse einige Nähte.

8. April. Verbandwechsel; Wrude sieht sehr gut aus. Jodoformgaze. Keine Schmerzhaftigkeit im rechten Arm mehr. Im mittleren Drittel des linken Oberschenkels stark schmerzende Anschwellung; Priessnitz. Temperatur $36,5-40,5$.

10. April. Furunkel der vorderen unteren Gehörgangswand incidirt. Verbandwechsel; Halswunde reactionslos. Schwellung und Schmerzhaftigkeit am linken Oberschenkel haben nachgelassen; Allgemeinbefinden sehr gut.

17. April. Senkungsinfiltration rom Proc. mastoideus nach der Halswunde zu. Aus der Wunde entleert sich auf Druck von oben rahmiger Eiter. Dilatation der Wunde und breite Incision der infiltrirten Partie. Tamponade mit Jodoformgaze. Schmerzen im linken Haftgelenk.

2. Mai. Das Fieber hat seit 5 Tagen vollständig aufgehört, die Schmerzhaftigkeit im linken Hüftgelenk besteht noch bei Druck auf dasselbe; die activen Bewegungen im Gelenk bei Räckenlage sind nicht behindert.

3. Mai. Die auf das linke Hüftgelenk bezogene Schmerzhaftigkeit ist, wie eine genane Untersuchung ergiebt, nur bedingt durch die geschwollenen linksseitigen Inguinaldrüsen.

22. Mai. Die Wunde am Halse ist fest vernarbt. Die Wunde hinter dem Ohr granulirt nur wenig.

2. Juni. Zurtickgebliebener Rest von adenoiden Wucherungen im Nasenrachenraum mit dem scharfen Löffel entfernt.

5. Juni. Klage über Schmerzen im rechten Ohr. Der hintere obere Theil des stark gerötheten Trommelfells ist vorgewölbt. Temperatur 39,7. 2 Blutegel.

7. Juni. Ausgesprochene Druckemptindlichkeit des rechten Proc. mast. Eisblase. Temperatur 39,1 .

8. Jani. Schwellung und Röthung hinter dem rechten Obr. Temperatur bleibt gleich hoch. Keine Senkung der hinteren oberen Gehörgangswand. Infiltration unterhalb des Warzenfortsatzes sehr schmerzhaft.

9. Juni. Oedem reicht bis zum Scheitel.

10. Juni. Durchbruch der hinteren oberen Gehörgangswand. Aufmeisselung rechts: Weichthelle stark infiltrirt. Corticalis unverändert. Nach den ersten Meisselschlägen quillt dicker, rahmiger Eiter aus dem Antrum mast. hervor. Tamponade. Eine Naht im oberen Wundwinkel. 
11. Juni. Temperatur wieder normal.

28. Juli. In poliklinische Weiterbehandlung entlassen.

15. August. Das rechte $\mathrm{Ohr}$ trocken; die Operationswunde verheilt. Anfang September auch das linke Ohr vollkommen geheilt.

Epikritische Betrachtung des Falles:

Obiger Fall bietet so manches Interessante, dass es sich verlohnt, auf einige Punkte noch etwas näher einzugehen. Zunächst müssen wir festhalten, dass die schwere Mittelohrentzitindung mit der consecutiven eitrigen Sinusphlebitis sich so unmittelbar an einen therapeutischen Eingriff unsererseits (Löffeloperation) anschloss, dass wir den Gedanken eines ursächlichen Zusammenhanges $z$ wischen beiden nicht zurückzuweisen vermögen.

Dass in Aubetracht der Unmöglichkeit, bei operativen Eingriffen im Nasenrachenraum streng aseptisch zu verfahren, hin und wieder acute Mittelohrentzündungen mit Ausgang in Eiterung nach der Operation der adenoiden Vegetationen vorkommen, ist nichts Aussergewöhnliches. Wohl aber ist es ein gewiss seltenes Eroigniss, dass diese secundären Entzündungen einen so bösartigen Charakter zeigen, wie in unserem Falle. Trotz der anerkannt zweckmässigen und möglichst frübzeitigen Behandlung der acuten Entzitndung sehen wir in wenigen Tagen eine Propagation des entzindlichen Processes eintreten, welcher nicht einmal in den Warzenfortsatzzellen Halt macht, sondern sogleich den benachbarten Hirnsinus ergreift. Ob der Entzündungserreger in unserem Falle ein hervorragend bösartiger gewesen, oder ob eine abnorm geringe Widerstandsfähigkeit der Kranken die Ursache dieses geradezu foudroyanten Verlaufes war, muss als offene Frage bezeichnet werden. Bacteriologiseh ist der Fall nicht untersucht worden, und irgend welche constitutionellen Ursachen der Bösartigkeit konnten, abgesehen von der hochgradigen Anämie der Kranken, nicht erwiesen werden. Immerhin ist es interessant, dass sich unter unseren Augen derselbe Process auf der anderen Seite wiederholte, nur dass es hier nicht bis zu einer Sinuserkrankung kam.

Dass wir zuerst (29. März) uns auf die typische Aufmeisselung des Warzenfortsatzes beschränkten, kann bei dem Fehlen des klinischen Bildes der Pyaemia ex otitide in der Zeit bis zu der Mastoidoperation nicht weiter Wunder nehmen. Das einzige bis dahin verhandene Symptom, welches überhaupt den Verdacht, dass eine Sinusphlebitis vorhanden oder wenigstens im Anzuge sei, hätte erregen können, war die Druckschmerzhaftigkeit im 
Verlauf der Vena jugularis. Dieses Symptom bat indess nur dann einen diagnostischen Werth, wenn es mit anderen für das Bestehen einer Pyämie sprechenden Erscheinungen vereint anftritt. Schwartze hat mit Nachdruck auf die Unzuverlässigkeit, die Druckempfindlichkeit im Verlauf der Vena jugularis diagnostisch zu verwerthen, hingewiesen 1) und betont, dass er wiederholt Gelegenheit hatte, in Fällen, wo eine ausgesprochene Druckempfindlichkeit in vita bestand, in autopsia das Feblen jeder Spur von Venenentzündung zu constatiren. Doch nicht nur die von Schwartze erwähnte Entzündung des Lymphplexus im Verlauf der Vena jugularis kann jene Erscheinung der Druckempfindlichkeit veranlassen, sondern unter Umständen anch andere Momente. ${ }^{2}$ )

Weiterhin liess uns auch nicht der Operationsbefund bei der Aufmeisselung auf den Gedanken kommen, dass eine Sinuserkrankung vorliege. Wir fanden keine äussere Wegleitung, etwa in Gestalt einer Fistel, welche den Suleus transversus perforirt hätte, ein Befund, der unter Umständen die Diagnose der Sinuserkrankung sehr zu erleichtern vermag.

Erst nach der Mastoidoperation, als das hohe Fieber fortbestand, sich auch Zeichen beginnender Metastasen (Infiltration am linken Oberschenkel u. s. w.) einstellten, und schliesslich am 4. April ein Schüttelfrost von balbstündiger Daner auftrat, ersehien die Diagnose der eitrigen Sinusphlebitis gesichert; und wie berechtigt unsere Indication zu dem zweiten operativen Eingriff war, das hat der Operationsbefund selbst bestätigt.

Die Art der Ausführung der Jugularisunterbindung mit folgender Durchsputlung ist eingehend in der Krankengeschichte geschildert. Hier soll nur noeh betont werden, dass der zur Durchspiulung des thrombosirten Gebietes angewandte Druck genau abgemessen werden musste; denn einerseits musste er die zum Fortschwemmen der Thrombenmassen, soweit sie eitrig zerfallen waren, nothwendige Stärke haben, andererseits musste er so schwach sein, dass er die noch nicht eitrig zerfallenen Massen, welehe peripherwärts von der Incisionsstelle des Sinus transv. einen soliden Wall bildeten, sowie auch die festen throm-

1) Handbuch der Obrenheilkunde, Bd. II. S. 847 .

2) Im vorigen Jahre hatten wir Gelegenheit, als Ursache einer ausgesprochenen Druckempfindlichkeit im Verlanf der Vena jugul, eine vom Schläfenbein ansgehende, in die Gegend des Kiefergelenkes hinabreichende Eitersenkung zu beobachten. 
botischen Abschlüsse an den Mündungsstellen der in den Sinus einlaufenden venösen Blutbahnen intact liess.

Die bisher noch nicht bei Unterbindung der Vena jugularis angewandte Durchspilung des in Betracht kommenden Theiles der venösen Blutbahn wird, wenn man bei der Abmessung der Druckstärke vorsichtig ist, kaum irgend welchen Schaden bringen können, bietet aber den grossen Vortheil, dass die eitrigen Thrombenmassen gründlich entleert werden und somit wohl zweifellos ein Fortschreiten des eitrigen Zerfalles der weiter peripher gelegenen noch soliden Thromben hintangebalten wird. Wir haben uns versehiedentlich in unserer Klinik bei der operativen Behandlung der eitrigen Sinusphlebitis auf die Freilegung, die Incision und einfache Ausspiilung des Sinus beschränkt, aber stets dabei gesehen, dass der Abfluss des Eiters aus dem Sinus ein nur sehr unvollkommener war. Diesem Uebelstande kann am besten durch die Durchspulung abgeholfen werden, deren Ausfubrung natürlich nur dann möglich ist, wenn die eitrige Schmelzung dex Thromben centralwärts bereits das Foramen jugulare iiberschritten hat.

Was nun den Einfluss unseres operativen Eingriffes auf den weiteren Krankheitsverlauf in unserem Falle anbetrifft, so können mindestens so viel behaupten, dass wir der Kranken nicht geschadet haben. Die Kranke ist vollkommen geheilt, d. b. nicht nur ihre Pyämie ist in Heilung übergegangen, sondern anch das ursächliche Ohrleiden; die Perforation im Trommelfell zeigt keinerlei Entzïndungserscheinungen. Wenn wir nun auch bei dem bekannten Vorkommen von Spontanheilungen bei der eitrigen Sinusphlebitis in unserem Falle nicht den Ausgang in Heilung einwandsfrei unserem therapeutischen Eingriffe zu Gute rechnen diurfen, so können wir uns doch des Gedankens nicht erwehren, gerade in diesem Falle, der einen so bösartigen Charakter von Anfang an zeigte, durch den Eingriff dem Weiterfortschreiten des Eiterungsprocesses Einhalt gethan zu haben.

Was die Erscheinungen nach der Unterbindung der Jugularvene anbetrifft, so ist Folgendes zu bemerken:

1. Ein weiterer Schuttelfrost ist in unserem Falle nicht aufgetreten.

2. Metastasen in anderen Körperregionen sind nach der Unterbindung nicht beobachtet. Die am 3. Mai, also 4 Wochen nach der Unterbindung, in der Krankengeschichte notirte Empfindlichkeit im linken Hiuftgelenk erwies sich bei genanerer Unter- 
suchung als eine irrtitmliche. Die Schmerzhaftigkeit sass niebt im Gelenk, sondern war bedingt durch eine entzindliche Sehwellung der Inguinaldrüsen, welche eine secundäre, durch das Infiltrat am linken Oberschenkel bedingte war. Letzteres bestand bereits oder war wenigstens im Entstehen schon vor der Jugularisunterbindung (vgl. Krankengeschichte).

3. Nach der Unterbindung bestand noch etwa 4 Wochen lang Fieber. Es ist dies eine Erscheinung, welche, soweit ich die Literatur kenne, in allen anderen nach der Jugularisunterbin. dung in Heilung übergegangenen Fällen ebenfalls beobachtet wurde. Was die Ursache dieses Fiebers anbetrifft, so glaube ich nicht, dass es in unserem Falle bedingt gewesen ist durch weitere Resorption pyrogener Stoffe von dem primären Krankheitsherde, sondern dass vielmehr zwei andere ursächliche Momente in Betracht kommen: einmal die durch die Blutbahn zur Zeit der Unterbindung bereits im Körper abgelagerten Mikroorganismendepots, welche zu den beobachteten Metastasen 1) Veranlassung gaben (linker Oberschenkel, rechtes Handgelenk, rechtes Schultergelenk, ferner auch die Entzündung der linksseitigen Inguinaldrisen, der Gehörgangsfurunkel), und zweitens die 12 Tage nach der Unterbindung eintretende Senkungsinfiltration vom Proc. mast. nach der Halswunde zu.

Wenn wir die in der Literatur bisher publicirten Fälle unserem hinzurechnen, so haben wir im Ganzen 9 Fälle von Unterbindung der Vena jugularis, darunter 7 mit Ausgang in Heilung. Trotz dieser sehr ermunternden Thatsache darf man nicht vergessen, dass trotz der Elimination des Krankheitsherdes rom Blutkreislauf durch die Jngularisunterbindung ein günstiger Ausgang nur dann za erwarten ist, wenn der Körper mit dem bereits aufgenommenen infectiösen Material fertig wird. Und wenn wir anch nicht in der Lage sind, den Krankheitsherd mit Sicherheit zu eliminiren, weil die Thrombenbildung nach vorn bis in den Sinus cavernosus und nach der anderen Seite hin den von der Jugularisunterbindung zu erwartenden Erfolg zu vereiteln vermag, weil dann eine Verschleppung von Thromben auf dem Wege der Vena jugularis der anderen Seite möglich ist, so glaube ich doch, dass gerade die gründliche Entfernung der eitrig zerfallenen thrombotischen Massen durch die Durehspülung des

1) Von Metastasen müssen wir hier reden, auch wenn ein Ausgang der Infiltrate in Eiterung nicht beobachtet wurde. 
78 III. GRUNERT, Geheilter Fall von Pyaemia ex otitide u. s. w.

in Frage kommenden Theiles der venösen Blutbahn am besten die Propagation der Thrombose oder wenigstens des eitrigen Zerfalles der Thromben hintanzubalten vermag. Immerhin wird die Erfahrung des oben geschilderten Falles mit dazu beitragen, in kommenden einschlägigen Fällen eine gleiche Therapie einzuschlagen: wir verbessern dem Kranken mit Wahrscheinlichkeit die Chancen auf Heilung, ohne ihn nach den bisherigen Erfahrungen durch den Eingriff selbst in Gefahren zu bringen, die grösser sind, als das Leiden selbst, um dessentwillen wir ihn operiren. 